\title{
Cognitive Behavioural Therapy Extended to Belief Background Therapy in Relation to Pakistani Cultural Antecedents
}

\author{
Dr. Mona Aeysha Khalid \\ Panasastra University of Cambodia; Email: mei3na4@gmail.com
}

Doi:10.5901/ajis.2015.v4n3p19

\begin{abstract}
It is an analytical review paper that conforms many strategies of CBT in various mental disorders: depression, anxiety, insomnia, phobia, BPD and anger management procedures. It is hypothesized that certain stresses are the product of certain cultures that need to be replaced by positive thoughts by utilizing a belief background therapy (a new therapy hypothesized and verified in this paper). It is also assumed that we perceive our environment, culture and experiences, in a particular moment that would keep on changing with new scenarios and settings in our belief backgrounds. Therefore, to change our behaviour (emotional and physical); we need to change our thoughts, beliefs and conduct - that would ultimately lead to changes in our belief backgrounds. Specifically, in the cases of stress, depression, and anxiety; belief background therapy needs to be addressed seriously and persistently. In the paper, we are, by choice, leaving healthy and helpful beliefs aside to avoid distractions and to keep the focus on the subject. The information collected through surveys, interviews and observations, of Pakistani females of average age- 40 years, suffering from some mental disorders- depression, anxiety, insomnia, phobia and stress. Mostly, females were dissatisfied through traditional methodologies of CBT and have left it in-between without feeling any progress at all.
\end{abstract}

Keywords: beliefs, stress, anxiety, depression, background, therapy

\section{Introduction}

CBT is based on an educational model. It is based on the assumption that all behavioural reactions are learned therefore it aims at unlearn the behaviours that the client wants to get rid of. This is not based on talking only; rather provides the client with an opportunity to learn new ways of dealing with his problems, identify new angles to think about it, continue practicing new techniques until he feels better and well (Hofmann, 2006, p. 26). This is the most important trait of CBT as people learn lots of patterns of thoughts from their immediate environment and culture; they find it hard to leave their old beliefs and think they are right in feeling whatever they feel (Dobson, 2009).

The second important trait of CBT is that its techniques rely on the inductive methods. It means it is based on the facts, not assumptions. Usually people make assumptions and believe them to be true. When they find that there is no need to be worried about self created worries (assumptions); they feel better. As in the inductive method (Free, 2007), we make a hypothesis that can be proved right or wrong as well. The client is ready to test his self created hypothesis and after testing its validity in the real world; he begins to think about changing his thinking according to the new information and understanding of the situation.

The third important trait of CBT is that it uses the Socratic Method (Cayoun, 2011) in which therapist tries to gain a deep understanding of clients' concerns. They usually focus on questioning and encourage the same method for the clients themselves. When a client question himself if he is right in thinking so and so, he is probably in doubt and finds the new understanding of his rigid belief he is sticking to. This is how a therapist finds out the real cause factors to diagnose or treat clients' problems accordingly (Dobson, 2010, 2011).

In magnification/minimization/catastrophizing, the client exaggerates the importance of things or inappropriately shrinks them-also called 'binocular trick'. To prove his/her point, a client is unconsciously focussing on others achievements and his failures. He is very much impressed by some areas/qualities/gains/ benefits/ successes and undermines his/her own personality potentials, abilities, and achievements as well. Not only this, sometimes client focuses on one negative event so seriously that he feels depression and anxiety symptoms all the time while ignoring other positive events and ignore them till the level they simply do not exist (Ronen, 2011; Antony \& others, 2005, p. 83)).

'Antecedent' is the events that occur before a behaviour followed by emotional and physiological reactions. Antecedents might be anything in the environment, any cognitive change, any emotional change, any event triggering 
sensations and perceptions of interpersonal relationships or any related event that either increase or decrease a specific response in client's emotional, cognitive and physical life; and these are especially important in assessing educational issues among adolescents (Reinecke \& others, 2003, p. 282). CBT counsellors can ask specific questions to find about antecedents such as:

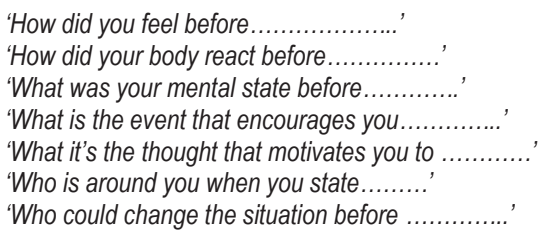

Automatic thoughts are combination of our instant thinking that generate intense emotions in us while experiencing a specific event or meeting with someone special. These thoughts should be comprised of conscious or unconscious beliefs, perceptions, sensations, or any kind of visualization we unconsciously believe in. They are spontaneous, many times illogical, self-created, learnt, and hard to ignore. We usually use the words 'ought', 'must', 'should', for such thoughts. These thoughts help us in making judgments, likes, dislikes, evaluations, and are enough to modify our behaviour ( risky, selfish, destructive, fearful, shaky). Usually how we perceive the situation around us is based upon our automotive thoughts processes. Cognitive behaviourists usually try to replace the negative automatic thoughts patterns with the positive ones (Cayoun, 2011)

Cognitive Distortions are some thinking styles that are unhelpful, distracting and highly negative that make a person vulnerable to a large number of psychological disorders and provide a big hindrance to get a changed new style of thinking that would lead to a positive behavioural change (Antony \& others $, 2005, p .83$ ). These distortions include: all or nothing mental filter, over generalization, disqualifying the positive, jumping to the conclusion, magnification, emotional reasoning, emphasis on should/must, labelling, and personalisation.

Most of the cognitive problems, like phobias, sexual preoccupations, anxiety attacks, traumatic memories, unwanted and unpleasant thoughts create emotional stress, thus it is very important to stop them. Thought-stopping techniques prove very useful when we are overwhelmed by our worries, fears, and are not able to stop our anxiety provoking thoughts (Marlatt \& Donovan, 2005). At first, in this technique, we have to identify/list three or four, the most troublesome thoughts among others. Then, we will also note/write down the most pleasing events or experiences of life. Our aim is to replace the negative thoughts into the positive ones. A 'thought log' is a table in which we can record our thoughts and replace them with pleasant thoughts. The therapist can consider some of the negative thoughts and replace them with pleasant thoughts and encourage the same technique in real life. Instead of a pleasant thought, anything else like saying 'no', touching your band, counting, singing a song, or taking a long breath will help to stop the unwanted thoughts. Moreover, the client can also consider a scheduled worry time to indulge upon his/her worries instead of worrying all the time for the same matter.

However, it is hypothesized that our automatic thoughts themselves are not considered as the only source of the problem; rather they are the indication of some problem in the background in some cases. When someone is having automatic thoughts like 'he is going to have a bad time or he should not go for this opportunity or he is in putting himself in danger by doing so etc.'; so to replace the thoughts alone will not solve the problem. Instead we need to deal with the cause of the problem behind the surface. If someone is having negative automatic thoughts whenever he meets someone new or start something new; he should look for the beliefs that generate such thoughts. He should analyze his emotional background under which influence, he is having such automatic thoughts (Heys, 2008).

Core beliefs are those 'statements' that we have faith in, consider a prerequisite for our decision making, developed through childhood experiences and nurturing techniques, make our identity, perception of the world around us, and are those concepts that remain within us to evaluate things, people and other events throughout our life span. Usually we unconsciously act upon our core-beliefs and find it very hard to challenge them. We follow values that are aligning with our core beliefs and then regulate our behaviour accordingly. These beliefs have the strong impact on our automatic thoughts (Hofmann, 2012, p.30). We can also be totally ignorant of our core beliefs and other times we can consciously choose our beliefs to follow strictly. In both cases, our core beliefs make our identity, personalities and behaviour overall that we would never be able to challenge unless we are willing to do so. Though it is a hard job to deal with our core beliefs; yet it is quite an achievable task and fulfilling duty to perform if it is for some goodness and healthy aim. Mostly cognitive psychologists try to deal with maladaptive core beliefs, just like negative automatic thoughts through 
a systematic procedure of questioning (Hofmann, 2012, p.30). However, not all kinds of core beliefs are responsible for one specific thinking error. When one is experiencing something strange on the daily basis; he or she needs to investigate his or her background beliefs (Hays, 2013) that are contributing towards his or her maladaptive behaviour at certain times. Such beliefs are part of one's background (emotional unconscious) and are solely responsible for making one depressed, obsessed or stressed in a particular way.

\subsection{Method}

In this research paper, various studies related to CBT have been investigated to reach a final and trust worthy solution to human behavioural aspects. We use a descriptive design to organize the related literature meaningfully and analyze carefully. In the end, a new hypothesis developed through both literature review and related data collected from 40 interviewees, has been proposed for further studies.

The main reasons to select the descriptive design is the complexity of the subject matter, and the unique nature of the purpose of the study, along with a high score of biased opinions when we use survey design on such subjects. In addition, it is not appropriate to trust only on instrumental techniques where subjects change their mind thousand times in a day. However, to prove this assumption, we have also interviewed certain individuals who also confide the cause of the study.

\subsection{Objectives}

The objectives of the study are:

a. To evaluate if our behaviour depends on our unique perception of our environment at any particular moment.

b. To predict that our behaviour depends on our unique belief background.

c. To analyse if our behaviour is subject to change due to having change in environment, thoughts, physical health and other factors.

d. To discuss the strategies to control our behaviour systematically through bringing a change in the unconscious emotional background in a particular situation.

e. To investigate if the unhealthy beliefs cause depression, anxiety and stress among vulnerable individuals.

\subsection{Sample}

In the typical society of Pakistan, 40 women diagnosed as depressed, phobic, patients of insomnia and anxiety have been selected for interviews. Each interview lasted for about one hour and the information about the subjects was also collected from their psychiatrists and relatives. The average age of the participants was 40 . Some information is also based on researcher's keen observation in a natural setting where these women live.

\subsection{Hypothesis}

It is hypothesized that our behaviour is dependent on our unique perception of the situation; unique belief background; and depends on our unique environment we are in at some particular moment. It keeps on changing across time, with changing background beliefs, and due to change in the immediate environment. To control our behaviour, we need to control our beliefs that act as unconscious emotional background in a particular situation. Thus it would be pertinent to study all types of background beliefs that could possibly damage our control on our behaviour. It is further hypothesized that in most of the cases of stress, depression, and anxiety, belief backgrounds had been easily over looked/ignored by the researchers and psychologists. However, in this study we will only consider unhealthy and miserable background beliefs that are more likely to put our integrity and self in trouble.

\section{Literature Review}

The two schools of thought named cognitive psychology and behavioural psychology, work together to form CBT. CBT is basically problem-solving focused therapy. It aims to alter thinking patterns within patients to activate positive change in behaviour. Cognitive psychologists study the cognitive areas (like thought processing, problem solving, perception, attention, memory and language) scientifically and have established framework in areas: schema theory, information 
processing model, and neuroscience model. CBT also deals with cognitive areas (negative perceptions of the world, negative beliefs, negative automatic thoughts, and negative concepts) termed as automatic thoughts.

CBT not only focuses on present situation; rather it focuses on the change in the behaviour (Beck, 2011). When we study the history of behavioural psychology, we come to know that John B. Watson in 1900s founded behaviourism, stating that only observable behaviour should be scientifically studied. Then B.F. Skinner followed the same path, stating how the behaviour is learnt through strengthening the behaviour and getting extinction through ignoring it. Therefore, through operant and classical conditioning, behaviourist psychologists aim at the change in behaviour. In other words, it means a change in the perception or a cognitive schema that brings a change in behaviour that can also be observed.

Thus, both schools of thought establish a background for CBT to apply the techniques of both ones to make the patients capable of changing their thought patterns and make them able to take the steps to bring a change in their lives. Beck, in his book Oxford Guide to Metaphors in CBT, described CBT as a "set of therapeutic interventions that aims to help patients learn to solve current problems and change dysfunctional thinking patterns that contribute to maladaptive emotions and behaviours (Ronen, 2011, p. 19)

Thoughts are usually a combination of beliefs, concepts, and perceptions about the world outside as well as inside. Beliefs are usually considered one of the major detriments of emotions. In cognitive-emotive theory, emotions result from how the individual believes the world to be (a point emphasized by appraisal theory).

In CBT, thought processes are divided into different types of schemas: person schema, role schema, scripts, content free and self-schema. In person schema, we feel about people being friendly, intelligent, nice or shaky. In roleschema, we perceive the groups and situations differently, thus feel according to the role of the persons. In scripts, we feel according to the events. For example, in party, we feel good, cheerful and pleasant whereas in church, we feel composed, indifferent and in peace. In content free schemas, feelings for the other person change due to our own perceptual tendency. And in self schemas, we feel according to our own self knowledge of our personality: being openminded, being strong, and being resilient, loving, caring, kind, powerful, crazy and funny. Such cognitions named as "automatic thoughts" are based on basic, general, core beliefs, called schemas (or schemata) that the person keeps about oneself, about the world, and the future (Hofmann, 2012, p. 2)

\subsection{Theories of Emotions}

Each theory of emotion brings a great amount of knowledge that we could apply to solve our daily emotional set-backs. However, to me, Cannon-Bard's theory is more realistic and applicable. As James-Lange theory focuses on physiological activation leading to the various conscious emotional experiences. It is not always true. Sometimes, we experience physiological dangers due to our unhealthy life style- not due to emotional reasons. Other times, our physiological changes are not so obvious to note or recognize fully in terms of emotions. Moreover, a large number of emotions create the similar changes in body that make it hard to interpret them and relate them with some specific emotion.

In Schachter \& Singer's two factor theory, emotions depend on two factors: autonomous arousal and cognitive interpretation of that arousal. In most of the situations, people do not look for the external environment to explain their emotion; rather rely on their autonomous arousal. However, in Cannon-Bard theory, physiological arousal occurs at the same time as emotional experience takes place. It is also proved scientifically that we cannot distinguish between our emotions (when they occur). How can we express them while we interpret our emotions at the same time we feel them physiologically and recognize them through cognitive schemas (King, 2001)?

\subsection{The Process of Change}

In the process of change, usually patients go through the following stages as per Hofmann (2012, p. 142):

a. Pre-contemplative

In the first phase of change, we are not consciously aware of the reason for a change. We believe that our lifestyle is perfect and there is no need to change it. We continue with our life as usual for a long time while keeping our bad habits with us.

\section{b. Contemplative}

In the second stage, we notice some signs of unhealthy routine to be worried about them. We in the beginning try to ignore them, while knowing that the situation is not as normal as it should be. Still we are not completely ready to think about the change we must bring in our life as early as possible.

c. Preparing: 
In the third place, we are completely aware of the fact that the change is now necessary, can't be ignored anymore. At this stage, we try to find out ways through what, we could adopt change strategies to help us.

d. Action:

Then eventually, we take action to bring a change. We adopt different strategies and are determined to face the resistance from time to time.

e. Maintenance

In the next stage, usually after 6 months, we have actually changed ourselves and never think of going back.

f. Termination

Then after years, we no longer are having any craving or likeness for our old life style.

Though, the procedure mentioned above has proved successful in most of the case studies, for most of the obsessive compulsive disorders treatments and for most of the phobias; however, it is strongly recommended to adapt a slightly different path for the people who are victim of negative or unhealthy beliefs. Say, a person is scared of darkness and could not even stay there for a second or so. He, not only needs a step-by-step exposure to darkness; rather he needs a replacement of beliefs that are associated with his phobia.

He might think that darkness would bring some aliens/ghosts/supernatural things in his way and could harm him. In this case his beliefs would need to be replaced by beliefs like: darkness brings calm, peace and tranquillity to brains. Darkness brings angels with pure blessings, as we close eyes to pray to find the same darkness. Darkness would make you brave and resilient. And many other beliefs out there in which he might choose to suit his needs and requirement.

There are many stages at which one could go back and choose unhealthy choices even after finishing the therapy course with a psychiatrist. Thus, at any time, the patient would be open to addressing unhelpful lifestyle choices. Change is a process rather than an event in our life. Being with the process, we continue with our emotional, physical and psychological changes taking place all the time consciously or unconsciously. When we are in stage 1, for one problem/disease, at the same time we are in different stages of change for another need. Even for one problem, we can fall back at any time from other stages and go for unhealthy life style habits.

The more we are motivated and determined to bring a change, the chances are that we will never go back and continue on the spiral of change. However, maintenance stage is more critical and tricky one as we are practicing a new life-style on a daily basis that we are not used to with. We could quit easily though, due to our impatient attitude, being attractive towards other bad life-styles, having no support group, lack of resources to consume, less emotional strength, and weak immune system to deal with new physiological and emotional changes.

\subsection{What Freud and Maslow assumed?}

According to Freud, 'Id', belongs to our instincts, animalistic traits that follow the pleasure principle and seek immediate satisfaction. These urges usually become part of the unconscious brain. These include our desire to eat, sleep, go to the toilet, and have sex. 'Ego ' being a part of the conscious world involves cognitive and perceptual processes that may include: memory, problem solving, control, information-processing, conflict analysis, language, etc. it also deals with defence mechanism that helps us to manage Id urges and we wait for an appropriate time to fulfil our biological needs. Superego guides us according to our moral and ethical standards that we adopt while living in a particular culture for a long time. It is also a part of our conscious (Freud, 1927).

However, it is proposed in this paper that there is some background behind each-id, ego, and super ego that is a combination of some beliefs, experiences and related evaluations. When we feel like to fulfil our desire immediately; we fulfil it in the way we have been taught. Some would cheerfully and delightfully eat what they like and some would remain compose in doing the same. Similarly, when we solve any problems; we solve it while utilizing some beliefs that we have been taught to solve it. Our complete understanding of our problems depends on the background (beliefs about it) that we carry for so long. Then in the process of decision making, when superego helps us to decide what is wrong and right; we are subject to change it through a background belief system that also helps us in making any evaluation and conclusion.

According to 'Maslow' self-actualizing person is he who makes use of his potentials, creative powers, and utilizes his energies towards a common goal. In addition to taking care of his basic needs like food, home and safety, such individuals are fully capable of taking control on their lives and their aims are usually beneficial for the society as a whole. They keep higher level goals to follow and do not hesitate to face the challenges in life. They are exactly aware of their life's purpose and the way to achieve it. Such individuals are capable of taking charge of their lives and could bring a change at international level for the sake of humanity and self-satisfaction (Maslow, 2000).

In addition to that, we can safely predict that such individuals must have a healthy, strong and motivating belief 
system in their brain that act as a supporting background throughout their life and especially before making any decisionmaking.

\section{CBT with Other Psychological Disorders}

To understand the CBT structure thoroughly, we need to see how it is related to other psychological mental disorders.

\subsection{CBT and Depression}

Usually, depressed people are overwhelmed by their negative thoughts and cannot address their educational, social, and personal needs properly on a daily basis. Such persons experience various symptoms: depressed mood, loss of interest in life, increased fatigue, negative thoughts, indecisiveness, poor control on emotions, low physical health, less concentration, less ability to plan properly or complete the tasks, no organization and no focusing are the prominent traits. Such persons find it difficult to carry on with their daily life activities with pleasure and ease. They find it very difficult to follow their goals, aims and overall life objectives. They are less motivated to bring a change in themselves and blame to their circumstances and environmental factors. For children, the similar symptoms may be observed. It is stated that, "depressed child may be withdrawn and isolate himself. On the other hand, a depressed child who is irritable may have frequent conflicts with peers and siblings and even begin to get into fights at school. Similar behaviours could be seen in a child with bipolar disorder (Fristad \& Arnold, 2004, p. 44). It is also verified that "some components of CBT are problem solving, communication skills, anger management, activity scheduling, and cognitive restructuring (i.e., learning to substitute helpful ways of thinking for the hurtful ways of thinking common in depression" (Fristad \& Arnold, 2004, p. 139)

Through 'activity scheduling', both therapist and the client come to know about their progress on certain measures. It establishes a base line for the therapist to understand the client's present activities, helps the therapist to suggest new skills and engagements to involve; and feels the client responsible, busy and progressive at times. For both, client and the therapist, it also points out the pleasurable and mastery activities to rate and continue with persistence and regularity. In the next stage, through the base line knowledge, the client is directed towards more scheduled activities including pleasurable and mastery ones. Then schedules are revised according to the prediction so that the depressed client get involved, seek confidence and feel better.

\subsection{CBT and Anxiety}

As the anxiety patients usually can't control the physiological symptoms associated with their anxiety: heat-beat, trembling hands, sweating, shaking, irritability, feeling sick, rapid thoughts, difficulty breathing, excessive worry, and agitation. They need relaxation strategies to control the situation at hand. In this regard, relaxation training and visualization techniques help a lot. These are proved methods to relax the muscular tensions during stress and anxiety episodes. These techniques (for example, abdominal breathing and muscle relaxation) teach the client to relax their body that ultimately slows down the thought processes and deals with the irrational excessive fears.

Anxiety and depression are very common symptoms in most of the disorders; however relaxation techniques, breathing exercises, activity rescheduling and thought stopping procedures help the patients a lot in getting control over their symptoms for a limited time period only. When we talk about life time changes in behaviour; we cannot just rely on the techniques described above. Especially depression and anxiety provoking experiences need to be addressed more in terms of background beliefs. Suppose one is fighting with the severe depression associated with his present situation in life. In this case, the walk outside, listening music, deep mediation or yoga or rescheduling will never prove useful. He needs to understand the cause of unique assessment of the situation, he is experiencing right now. In most of the cases, presumed standards about the people, perfection, or life in general drag us towards depressive episodes of helplessness and hopelessness. Our core beliefs hinder our progress towards success and happiness.

\subsection{CBT and Phobia}

People with a specific phobia usually face lots of physiological and emotional distress on the exposure of phobic situation. They usually feel panic out of irrational fear, rapid heart beat, shortness of breath, trembling, shaking, sweating, dry mouth, palpitation, and other related symptoms. Relaxation training would help them to control their symptoms through abdominal breathing and progressive muscle relaxation. They can control their breathing, heart rate, and pulse through 
abdominal breathing at some specific phobia exposure. And for other symptoms, like shaking and trembling, the progressive muscle relaxation would help them to control the body and maintain a healthy balance. (Maj \& others, 2004)

The systematic desensitisation is a process in which a client with some phobia is exposed to a less threatening situation in the beginning of the process. Then his exposure is enhanced through a more dangerous situation and the process continues unless he is ready to face his real phobia with normal emotional response. Thus we divide the actual trigger into many small effects and try to make the client comfortable step by step (Maj \& others, 2004, p.180).

For example, someone has a social phobia and can't stay in a public gathering for a minimum five minutes. We can divide his fear into the following small tasks:

1. Imagine being in a gathering and feel calm.

2. Stay with your friends in a group for 30 minutes.

3. Go for a party of 5-10 people and enjoy.

4. Celebrate your birthday and invite 10-15 friends and enjoy.

5. Go for a public show for 5 minutes and relax yourself with your favourite music.

It is further hypothesized that phobia is linked back to some beliefs and related past experiences of life that generate anxiety symptoms for a patient of phobia. Only desensitization of some phobic situation will not work properly unless we deal with the root cause of the problem through enquiring a series of probing questions. Our fears are usually based on some justifications that we think are true and healthy. When we are taught about the unnatural and fake perception of our phobic situations; we are over it successfully. For example, a person scared of heights would be thinking like, 'heights are dangerous to health'. When we replace this belief by this, 'heights are safe if we are aware of safety issues', we are no more a phobic person. The same is true to other phobic situations that link to our core belief backgrounds.

\section{$3.4 \quad C B T$ and Insomnia}

Insomnia is a condition in which the client feels great difficulty in initiating, maintaining, and re-storing the sleep for at least one month. The sleep's quality is also poor enough to carry on the day to day functioning properly, continue with educational goals successfully or maintaining social relationships normally. The client never feels refreshed, no matter how much sleep he has been gone through. He feels that he is usually awake while lying on the bed for many hours in order to get a sound sleep. This type of sleeplessness is not related to another mental disorder /disease or another physiological effect of any substance taken (Antony \& others, 2002, p. 542).

The persons with insomnia often spend too much time in bed in the hope that they will fall asleep. They are advised to keep a sleep routine daily and then restrict themselves from going to bed unnecessarily. Their sleep timings are monitored and they are advised to go to bed only in that time span they are more likely to fall asleep. That is why this method is called 'sleep restriction' method. In this method, initially, a strict night routine, effective to the client is encouraged to maintain. He will go to bed later than the usual time and feel sleepy in the day time. Meanwhile, the time of sleep will be gradually increased and more restful sleep will be maintained. We can start with six hours schedule in the beginning and after having a good night sleep of this time span, we can include 30 minutes more and then 30 minutes more. By the end, eventually a 7 or 8 hours, nice, peaceful, and sound sleep could be achieved and maintained. However, the timings of the sleep may vary according to the specific nature of insomnia the client is suffering from (Antony \& others, 2002, p. 542).

Sleep restriction method along with cognitive therapy has proved a successful technique; however to use sleep hygiene alone is not very satisfactory tool for various patients (Antony \& others, 2002, p. 542). It can be further speculated that sleeplessness can also be due to various unhealthy background beliefs one carries about the sleep. Additionally, it can be an indirect symptom of some other disorder to deal with at first place. Last but not the least it can be due to some belief background that needs to be addressed first. For example one believes that, "success means many restless nights before" or one thinks that, "mother has to wake up many times a night for her child for safety concerns". Similarly there could be any thing that behaves like an unhealthy belief background for a person suffering from insomnia.

\subsection{CBT and Anger Management}

In the anger management procedure we need to know the following triggers and avoid them as much as possible.

1. Frustration: when someone is instructed for not doing something, frustration is manifested and put the person 
towards anger.

2. Annoyance: when distraction occurs while you are doing something seriously, you may become annoyed after sometime. This might feel angry if the situation is not resolved.

3. Mistreated: when someone is not treated fairly or ignored or feel discriminated, angry sentiments are essential to experience.

4. Abuse: any kind of physical, verbal, emotional, or physiological abuse leads us towards angry emotions.

5. Disappointment: when our needs, wishes, aspirations, and hopes are not fulfilled or are partially fulfilled, we feel disappointed that ends in making us angry over the situation.

To conclude, Cognitive behaviour therapies prove very useful and successful in managing anger, anxiety, depression and other control issues. Novaco's (1975) anger management approach can be utilized for some purposes to some clients (Dutton (1995, p.253).

However, there are still chances that one is under the influence of some unhealthy belief system. For example one might think that "one needs to be angry to convey the message firmly" or one might think that, "strong persons keep their anger inside" or one might think that "to get attention from others, one has to be angry" and there could be any statement of belief like that.

\section{DBT (Dialectical Behaviour Therapy)}

In the 1970s, Marsha M. Linehan noted that CBT, as the only method available to treat women with BPD, is not sufficient. She noted that in the traditional CBT, the treatment was directed by the client-comfort who is responsible for choosing strategies (either effective or ineffective) whereas in DBT, new strategies based on client-acceptance were introduced (Barlow, 2001). From the following list, we can see in how many ways, DBT is different to traditional CBT:

a. In CBT, the primary aim of the theory was to control suicidal tendencies and crisis related; whereas in DBT, the clients learn about their strength and skills to work on in the future.

b. In addition to CBT, in DBT, certain dialectic strategies were added that make the counsellor keep the balance between acceptance and change in each session. It encourages the client to become active, speed up recovery and move forward.

c. DBT is based upon an extended therapeutic frame work where as CBT stands for a small theory technique.

d. In contrast to CBT, DBT could be extended to one or more years; could involve individuals or groups and could include behaviour techniques to bring changes in client's life.

e. In the treatment of BPD, DBT utilizes modes, stages and targets and has been very successful based on extensive research procedures.

There could be many reasons for developing BDP in a person. The main one is that the child has been raised in an abusive, non-nurturing, fearful, and unhealthy environment where she is vulnerable to many types of emotional and physical stresses. This kind of vulnerability might have been linked to genetic causes; however in a non-nurturing culture, one becomes aware of the fact that if he/she wants to get attention to be accepted and to feel worthy; he/she might need to threat or harm him/her self. Especially in intimate relationships, patients of BPD express their negative feelings, anger, rejection and the hurt through harming themselves or through giving suicidal threats.

The patients with BPD often have difficulty in maintaining relationships through communication skills, and discussions. They feel vulnerable when they have to seek help from others, ask something, or disagree with someone. They can't keep a healthy balance between needs and wants, priorities vs. duties, rights and liabilities, being assertive and supportive etc. DBT provides such patients a frame work that encourages interpersonal effectiveness through relationship building techniques. Clients learn the new technique to express themselves, their needs, wants, desires, while observing limitations and boundaries. It encourages maintaining healthy relationships with effective communication skills while keeping mutual respect.

People with BDP often feel difficulty in regulating/controlling emotions/moods, become unpredictable and often cross limits in anger. Some BDP patients involve in avoidant behaviour such as substance abuse, self-harm or alcohol. DBT in this regard, teaches the clients about problem-solving, checking facts about situations, alternative responses, positive feedbacks, emotional vulnerability, and about feelings in depth to regulate emotions naturally.

BDP patients are usually susceptible to various damages, and destructions, or self harm. They try to escape from their unpleasant feelings and take impulsive actions; though, they feel guilt after some time on their impulsivity.

In DBT, clients are taught how to deal with their stress, control emotions, and tolerate distress effectively. It provides the client with many solutions to solve their problems, to deal with difficult emotions, and to control painful 
extremes in emotions, especially in a crisis situation. They learn to cope with their problems with various strategies rather than engaging in self-harm or destructive activities. In short "Dialectical behaviour therapy has been shown empirically to be a particularly effective treatment of BPD" (Widiger \& Mullins-Sweatt, 2008, p. 1734).

\section{SFBT (Solution Focused Brief Therapy)}

SFBT in contrast to CBT is more focused on clients' present and future instead of past problems. SFBT therapists believe that the clients being fully equipped by the potentials can solve their problems.

SFBT covers lots of areas like relationship problems, domestic violence, depression, anxiety, parenting issues, social problems, gambling, abuses of different kinds, and suicidal tendencies. Its focus is to work on solutions compared to solving problems. It is more concerned about strengths, past successes, and future success than past issues, problems, and their future links.

Both CBT and SFBT focus on learning new strategies; though SFBT stresses upon continuing with those strategies that have already been working. In SFBT, clients are being helped in selecting their path of actions towards a change whereas in CBT, clients are guided throughout step-by-step to bring a positive change in themselves.

Unlike CBT, SFBT motivates the client to set their own homework, keep driven and in spirit to complete the set tasks without having any pressure to finish them in time.

SFBT can be applied to various kinds of difficulties such as relationship problems, domestic violence matters, parent-child issues, intimate relationship issues, depression, anxiety, social relationship problems, gambling, abuses of various kinds (such as child abuse, verbal abuse, partner abuse, physical abuse, emotional abuse, physiological and psychological abuse), and other suicidal tendencies.

\section{BBT- Belief Background Therapy}

Have you ever encountered that your boyfriend is behaving differently with you in front of person 'a' or 'b'? Have you ever noticed that your son is indifferent to you while sitting in the company of other relatives/friends? Have you ever noticed someone you know from your heart, is behaving oddly in his office or at any other particular place? Have you ever argued with someone for stating two different contradictory statements at two different places? Have you ever thought people change their attitude due to their diplomatic mind-set? One for sure encounters such experiences at least once in his or her lifetime.

However, not all experiences are due to our misperceptions. Some of them are true examples of cheating, diplomacy, and duality. My point, in this regard, is that our backgrounds have a strong effect on our thoughts, actions, and attitudes and that (effect) is beyond our expectations. For example, one can show his face a very positive individual while sitting in his parents' company and much negative image of his self in his friends' company consciously or unconsciously. One can behave aggressively with his family members and at the same time act like a gentleman in his office with coworkers. It could also be the other way round. One can be a genius in front of his relatives and friends, and proves criminal under investigation by the police. There could be a thousand other examples to quote in this regard.

In fact, our backgrounds are our predecessors and predict our behaviour strongly. The main background categories include: information about self, thoughts about significant others, interpretation of the events happened, information about the place of experience, thoughts within unconscious about anything, beliefs about the information perceived and any kind of future association.

When we are born, we are given some messages by our parents, relatives and the culture we are in. we are not able to analyze them but we unconsciously download them from the world outside in our brain disk. For example a girl is conveyed some messages that include: I am a pretty girl, mom loves me so much, I am not supposed to disobey, I am not supposed to behave rudely, I am supposed to take care of my younger siblings, I am not as important as my male siblings are, I am a girl who is supposed to leave the parental home after marriage (a belief common in the society of Pakistan). These all beliefs about the gender role would play their role as a background when the girl will behave in a certain manner. In the same manner, beliefs about the other people and interpretation of the events act as a background when we need them. However, such beliefs are usually formed in the childhood age (from birth till puberty) and become quite rigid to deal with, in our adulthood.

The place of experience includes all related information about the place at which you are. For example, if you feel secure in your parents' home and love to be there, you will act naturally a nice person and speak gently unconsciously. On the other side, you will become angry and behave rudely in a place you simply do not want to go. 
Therefore, mostly people look calm, cool, and friendly at meditation places such as mosques, churches, yoga centres, temples and fitness clubs.

Thoughts in unconscious mind always dictate us and show us the way to behave. You know when you are going to the hospital in an emergency; you will become a little anxious, worried and depressed. You may drive rashly or slowly due to your unconscious thoughts going on. Oppositely, if you are going to marry, you will be cheerful, excited, and romantic in your behaviour and conduct. Your thoughts would also become more imaginative and innovative at that time.

Beliefs about the information perceived are the strongest part of us that guide us in each moment of life. When someone behaves nicely in front of his friends, he might have a belief that friends must be given priority over others in life or any other belief conveying the similar meanings. On the contrary, one can behave rudely with his wife based on the belief that one should act like a lion in front of his family.

The last background is future orientation or association in mind. We behave according to our expectations and priorities. When we meet someone we think could give us something in the form of money, respect, comfort, or anythingtangible or intangible; we behave good, wise, compose and even empathetic. Whereas with the person who we think could never be able to support us in any form or may harm us in one way or the other, we use other tactics to ignore him like showing indifferent, rude, uninterested or even apathetic emotion/attitude.

To bring a change in our belief background, we would need to follow a proper systematic procedure. It will all depend on our unique situation and concepts we carry along. However for clarity purposes, it is assumed that one could bring a change in his environment ( by changing his residence, office, leisure place, or any significant place he is associated to), in his identity ( by adopting any new thing, by starting a new job, by getting a new training course, by joining a forum ), in his perceptions ( by reading about other religions, by getting to know other belief systems, by interacting with people with different backgrounds, by having new friends) and a change in his or her future ( by planning kids, family, or holidays).

Thus, we change not only our attitude, rather behaviour and thoughts according to our needs ahead at any particular time. We unconsciously get information from our backgrounds about the place we are acting in, people we are interacting with, the scenario in which we are dealing with others and about the beliefs linked with those experiences. We calculate it all in the shortest possible period and then act accordingly. In this cognitive processing, we are likely to make mistakes; though others around us take it the other way round, as they are not aware of our background knowledge of the event. Such mistakes or misperceptions may cost a high price if not understood properly in time.

\section{7. $\quad$ Findings}

In our surveys, observations and interviews, it was the obvious fact that 90 percent patients of depression, 89 percent of anxiety, 79 percent of insomnia, 89 percent of phobia, 94 percent of poor control and 99 percent of borderline personality disorders were not completely happy with the procedures of CBT. Patients overall were suffering from various kinds of mental disorders due to their unhealthy cultural beliefs along with negative traditional set ups. Some of them wanted to bring a change in their lives but due to their cultural hurdles and having no support from family and friends, could not continue with their struggles.

The subjects proved that their behaviour is due to their perception of their environmental /cultural beliefs. They behave in the manner their culture expects them to be. They feel good if they behave according to the traditions of their society whether it is abusive in nature or not. $78 \%$ claimed that their actions depend on their unconscious mind (unique belief background) which means when they are mentally disturbed; they react in anger and behave abnormally. On the other hand, when they are happy inside, they behave better with others and become normal. Subjects also proved that they feel better in a different environment (the environment of their choice), with different people (the people they love), and whenever they are fit and healthy. Most of them ( about 60\%) claimed that they had tried to control their behaviour through bringing a change in their unconscious thought processes; however it did not work as they expected. It is also proved that some unhealthy beliefs were creating an environment of depression, anxiety and stress among the subjects interviewed in the present study.

\section{Recommendations}

In this paper, a new theory named belief background therapy has been established and proposed. However, it is strongly recommended to carry out a thorough research work on the reliability, validity and consistency of this theory in terms of its effectiveness and usability. 
This is particularly important for the people living in the typical culture of Pakistan. However, in other areas of the world, the same theory can be established and verified with new research design and cultural parameters.

As our sample consisted of female participants, the other researchers can target male participants as well to extend the implication and credibility of the research purposes.

Lastly, due to the critical emotional situation of Pakistani women in general (Mona, 2015), it is highly recommended to gather the information about the negative beliefs, depressing traditions, and unhealthy cultural teachings that create stress, anxiety and depression among women of Pakistan till the level they enter into the realm of mentally disordered population; and find out ways to tackle them to uplift the cognitive health of the women over all.

\section{References}

Antony, Marin M. \& Barlow, David H. (Eds). (2002). Handbook of assessment and treatment planning for psychological disorders. NY: The Guilford Press. P.544.

Antony, Martin M, Roth Ledley, Deborah, \& Heimberg, Richard G. (Eds). (2005). Improving outcomes and preventing relapse in cognitive behavioural therapy. NY: Guilford Press.

Barlow, David H. (Ed). (2001). Clinical Handbook of psychological disorders: A step-by-step treatment manual. NY: The Guilford Press. P. 477

Beck, J.S. (2011). Cognitive behavior therapy: Basic and beyond (2nd Ed). New York: Guilford.

Cayoun, Brouno A. (2011). Mindfulness-Integrated CBT: principles and practice. Malden, MA: John Wiley \& Sons, Ltd. P. 256.

Dutton, Donald G. (1995). The domestic assault of women: psychological and criminal justice perspectives. Vancouver, B.C.: University of British Columbia Press. P.253.

Dobson, D.J.G., \& Dobson, K.S. (2009). Evidence-based practice of cognitive behavior therapy. New York, NY: Guilford Press.

Dobson, K.S. (Ed). (2010). Handbook of cognitive-behavior therapies(3rd Ed). New York: Guilford.

Dobson, K.S. (2011). Cognitive therapy. Washington, DC: American Psychological Association.

Free, Michael L. (2007). Cognitive Therapy in groups guidelines and resources for practice. Hoboken, NJ: John Wiley \& Sons, Ltd.

Freud Sigmund. (1927). In Jones Ernest (Ed). The Ego And The Id. UK: Leonard \& Virginia Woolf.

Fristad, Mary A. and Goldberg Arnold, Jill S. (2004). Raising a moody child: How to cope with depression and bipolar disorder. NY: The Guilford Press.

Hofmann, Stefen G. ( 2012). An introduction to modern CBT psychological solutions to mental health problems. UK: John Wiley \& Sons, Ltd., Publication. Pp.26-30

Hays, P.A. (2013). Connecting across cultures: The helper's toolkit. Thousand Oaks, CA: Sage.

Hays, P.A. (2008). Addressing cultural complexities in practice: Assessment, diagnosis and therapy (2nd Ed). Washington, DC: APA

King M.G. (2001). Emotions in the workplace: biological correlates. In Roy L. Payne. \& Cary L. Cooper (Eds), Emotions at work, theory, research and applications in management. UK: John Wiley \& Sons Ltd. P. 89.

Maj Mario, Akiskal, Hagop S., Juan Jose Lopez-Ibor, \& Ahmad Okasha. ( Eds). (2004). Phobias. NJ: John Wiley \& Sons, Ltd. P.180.

Marlatt, G. Alan \& Donovan, Dennis M. (Eds). (2005). Relapse prevention: Maintenance strategies in the treatment of addictive behaviors. NY: Guilford Press. P.75

Maslow, Abraham H. (2000) In Deborah C. Stephens (Ed), The Maslow Business Reader. NY: John Wiley \& Sons.

Mona Aeysha, Khalid. (2015). Strategies to change beliefs that cause low self-esteem, depression and anxiety among women of Pakistan. Journal of Educational and Social Research. Vol 5. No(2). P.153

Reinecke, Mark A., Dattilio, Frank M., \& Freeman Arthur (Eds). (2003). Cognitive Therapy with children and adolescents: A casebook for clinical practice. NY: The Guilford press. P.282

Ronen Tammie. ( 2011 ). The Positive Power of Imagery. Harnessing Client Imagination in CBT and Related Therapies. UK: Wiley Black Well. P. 284.

Sexton, Thomas L.; Weeks, Gerald R., \& Robbins Michael S. (2003). Handbook of family therapy: the science and practice of working with families and couples. NY: Brunner-Routledge. P.104

Widiger, Thomas A. \& Mullins-Sweatt Stephanie N. (2008). In Tasman Allan; Kay Jerald; Lieberman, Jeffrey A.; First Michael B., \& Maj Mario. (Eds.). Psychiatry (3rd Ed). NJ: John Wiley. P.1734 
